\title{
Use of cyclosporin in the treatment of steroid resistant post-keratoplasty atopic sclerokeratitis
}

\author{
Mark D Daniell, John K G Dart, Susan Lightman
}

\begin{abstract}
Aims-To demonstrate that oral cyclosporin is successful in the management of steroid refractory post-keratoplasty atopic sclerokeratitis (PKAS). Methods-Series of three patients.

Results-The patients developed PKAS between 2 and 11 days after surgery. All cases failed to respond to oral prednisolone $60-80 \mathrm{mg}$ daily for $10-30$ days but resolved with the addition of systemic cyclosporin 5.0-7.5 mg/kg/day. There have been no relapses following withdrawal of therapy after 3-18 months.

Conclusions-PKAS is an uncommon, but severe, complication of corneal graft surgery and often responds to oral prednisolone treatment. Oral cyclosporin results in rapid resolution of PKAS in steroid unresponsive cases.

(Br f Ophthalmol 2001;85:91-92)
\end{abstract}

Penetrating keratoplasty may be required to treat keratoconus and corneal scarring associated with atopy related eye diseases. ${ }^{1}$ Intense inflammation can occur shortly after surgery resulting in wound dehiscence, failure of epithelialisation, and early rejection called post-keratoplasty atopic sclerokeratitis (PKAS). ${ }^{2}$ This condition occurs uncommonly particularly in atopic individuals with high $(>1000 \mathrm{IU} / \mathrm{ml})$ serum IgE. They present up to 10 days postoperatively with severe photophobia, pain, epiphora, mucus discharge, intense conjunctival and anterior scleral injection with mucus adherent to the sutures which can cheesewire through the inflamed tissues leading to wound slippage and frank dehiscence. Later, microbial keratitis and graft rejection

Corneal Service, Royal Victorian Eye and Ear Hospital, Melbourne, Australia

M D Daniell

Moorfields Eye

Hospital, City Road, London, EC1V 2PD, UK

J K G Dart

S Lightman

Institute of

Ophthalmology, Bath

Street, London

EC1V 9EL, UK

S Lightman

Correspondence to: John K G Dart

jdart@dial.pipex.com

Accepted for publication 5 July 2000 right $(8 \mathrm{~mm})$ penetrating keratoplasty was performed using 16 interrupted sutures and subconjunctival betnesol at the end of surgery.
The day after surgery there was minimal inflammation but at 4 days postoperatively, he developed acute inflammation, photophobia, and pain in the right eye and was diagnosed as having PKAS. Dexamethasone eye drops $0.1 \%$ were increased to hourly and prednisolone 80 mg daily was added. After 8 days (day 15 postoperatively), there was less pain but severe inflammation remained with anterior scleritis and infiltrates at the host insertion of some sutures. Oral cyclosporin (Sandimmun, Novartis), was added at $5 \mathrm{mg} / \mathrm{kg} /$ day. The inflammation completely resolved over the subsequent 12 days (by 27 days postoperatively) and the prednisolone was reduced over the following 3 months when it was stopped, followed by the cyclosporin. Two loose corneal sutures were removed and replaced but no further episodes of inflammation occurred. The graft was clear 2 years later.

CASE 2

This 20 year old man had a history that included asthma, eczema, and perennial allergic conjunctivitis. Keratoconus was diagnosed in 1985 and he underwent a complicated right penetrating keratoplasty in 1986. Acute hydrops developed in his left eye in 1987 leaving an axial scar that limited visual acuity with a contact lens to $6 / 60$ in 1993 when he was referred. His serum IgE was $120 \mathrm{IU} / 1$ before surgery.

A left $8.0 \mathrm{~mm}$ penetrating keratoplasty was performed using a continuous suture. The following day the graft was thickened with no epithelial cover and the conjunctiva was injected. Unpreserved dexamethasone $0.1 \%$ drops were started with antibiotic prophylaxis and although the graft started to epithelialise, at 11 days postoperatively there was anterior scleritis and infiltrates at the host suture insertions. A diagnosis of PKAS was made and prednisolone $60 \mathrm{~g}$ daily was commenced. After 4 days on this regimen (15 days postoperatively) the microinfiltrates had resolved and the cornea epithelialised but there was still substantial anterior scleritis and mucus attached to a loosening graft suture. The prednisolone dose was therefore increased to $80 \mathrm{mg}$ daily. After 6 days (now 21 days postoperatively), the inflammation was resolving but the graft edge had lifted over 8 clock hours. Cyclosporin (Sandimmun) was added at $5 \mathrm{mg} / \mathrm{kg} /$ day to try and improve control of the inflammation. After 14 days on this regimen (35 days postoperatively) the inflammation had almost resolved but the suture had cheesewired. The graft was resutured with interrupted sutures 42 days postoperatively but as the limbal inflammation had increased further, he was pulsed with intravenous methylprednisolone $500 \mathrm{mg}$ and the 
cyclosporin increased to $7.5 \mathrm{mg} / \mathrm{kg} /$ day. The inflammation subsided over the subsequent 7 days (57 days postoperatively) and both the prednisolone and cyclosporin were reduced. Three loose sutures were removed and 2 weeks later (86 days postoperatively) the inflammation had completely resolved. Both drugs were tapered slowly over the next few months with no further inflammation and he has since achieved 6/9 with a contact lens.

CASE 3

This 44 year old atopic man, with asthma and eczema, had left unilateral keratoconus for which a penetrating keratoplasty had been carried out in 1979 . He presented in 1994 with a failed left graft without a clear history of rejection. A repeat left penetrating keratoplasty (8 $\mathrm{mm}$ ) was performed (5 January 1995) using interrupted sutures. Five days postoperatively he returned to the casualty department with severe pain and a very inflamed eye. There was a moderate uveitis with a clear graft but severe anterior scleritis. PKAS was diagnosed and he was started on oral prednisolone $80 \mathrm{mg}$ daily and tapered to $60 \mathrm{mg}$ after 1 week. The inflammation was not controlled by 35 days postoperatively and cyclosporin (Sandimmun) was started at $5 \mathrm{mg} / \mathrm{kg}$ /day with oral prednisolone and intensive topical steroids. Fourteen days after starting this regimen (49 days postoperatively) his pain had resolved and there was no inflammation. The prednisolone was tailed off to $10 \mathrm{mg}$ oral daily over the next month and the cyclosporin slowly reduced and stopped. A low dose of oral prednisolone was continued for his eczema and 2 years later, he had a spectacle acuity of $6 / 12$ with a clear graft.

\section{Discussion}

PKAS is a syndrome seen in the early postoperative period in some atopic individuals who may have an elevated serum IgE. When untreated, graft failure, due to infection and rejection, is the normal outcome. PKAS is usually adequately suppressed by the administration of a prolonged course of high dose oral and topical steroids with good long term results. ${ }^{2}$ This report describes a group of three patients with PKAS that was refractory to the use of high dose oral and topical steroids alone but which responded to the additional use of oral cyclosporin. This combined steroid and cyclosporin therapy suppressed the inflammatory response and resulted in resolution without recurrence after withdrawal of systemic therapy.

The pathogenesis of this form of sclerokeratitis is unclear. All these patients are atopic, usually with an elevated serum IgE, and often have or have had conjunctival inflammation associated with atopy. Many different types of cells are present in the atopic conjunctiva including $T$ cells, macrophages, mast cells, and eosinophils, ${ }^{3}$ all of which can produce a variety of cytokines as can the conjunctival epithelium. ${ }^{4}$ Most types of inflammation, whether $\mathrm{T}$ cell or mast cell initiated, are usually downregulated by corticosteroids but immune mediated disease which is refractory to steroids and responds to cyclosporin is well documented in the literature..$^{56}$ Although steroids will downregulate most cytokine production, tumour necrosis factor alpha (TNF- $\alpha)$, which is produced early in graft rejection ${ }^{7}$ as well as by conjunctival epithelial cells, ${ }^{4}$ has been shown to modulate the effects of steroids on human monocytes so that they become refractory. ${ }^{8}$ In addition, these patients were atopic and it has been shown in allergic conjunctival disease that eosinophils ${ }^{9}$ and $\mathrm{T}$ cells are present ${ }^{3}$ and able to secrete a variety of cytokines including IL-5. It is known that cyclosporin, but not steroid, is able to inhibit IL-5 enhanced granule release and maybe selected cytokine from eosinophils, whereas steroids cannot. ${ }^{10}$ It is therefore possible that one or all of these mechanisms are contributing to the rapid response to cyclosporin when steroids had not been effective in these patients.

Systemic steroid prophylaxis is not currently suggested routinely for this group of patients as it is a rare complication and many patients with allergic disease undergo successful corneal grafting without developing PKAS. In high risk patients, who have had PKAS after previous grafts, prophylaxis may have a role. Routine prophylactic use of cyclosporin ${ }^{11}$ is not justified as most atopic patients will be unaffected by PKAS and cyclosporin carries significant additional risks including future malignancy.

1 Castroviejo R. Keratoplasty in treatment of keratoconus. Arch Ophthalmol 1949;42:776-800.

2 Lyons CJ, Dart JKG, Aclimandos WA, et al. Scleritis after keratoplasty in atopy. Ophthalmology 1990;97:729-33.

3 Metz DP, Hingorani M, Calder VL, et al. T-cell cytokines in chronic allergic eye disease. F Allergy Clin Immunol 1997;100:817-24

4 Hingorani M, Calder VL, Buckley RJ, et al. The role of conjunctival epithelial cells in chronic ocular allergic disease. Exp Eye Res 1998;67:491-500

5 Fernandes NF, Redeker AG, Vierling JM, et al. Cyclosporine therapy in patients with steroid resistant autoimmune hepatitis. Am $\mathcal{F}$ Gastroenterol 1999;94:241-8.

6 Garcia C, Michelon T, Barros V, et al. Cyclosporine in the treatment of steroid-dependent and steroid-resistant idiopathic nephrotic syndrome in children. Transplant Proc 1998;30:4156-7.

7 Zhu S, Dekaris I, Duncker G, et al. Early expression of proinflammatory cytokines interleukin-1 and tumor necrosis factor-alpha after corneal transplantation. F Interferon Cytokine Res 1999;19:661-9.

8 Franchimont D, Martens H, Hagelstein MT, et al. Tumor necrosis factor alpha decreases, and interleukin-10 increases, the sensitivity of human monocytes to dexamethasone: potential regulation of the glucocorticoid receptor. F Clin Endocrinol Metab 1999;84:2834-9.

9 Hingorani M, Calder V, Jolly G, et al. Eosinophil surface antigen expression and cytokine production vary in different ocular allergic diseases. $\mathcal{F}$ Allergy Clin Immunol 1998;102:821-30.

10 Meng Q, Ying S, Corrigan CJ, et al. Effects of rapamycin, cyclosporin A, and dexamethasone on interleukin 5 -induced eosinophil degranulation and prolonged survival. Allergy 1997;52:1095-101.

11 Reinhard T, Sundmacher R. Perforating keratoplasty in endogenous eczema. An indication for systemic cyclosporin A. Klin Monatsbl Augenheilkd 1992;201:159-63. 